\title{
Reverse osmosis membrane modified by interfacial polymerization in non-polar heptane solvent assistance with acetone as a co-solvent
}

\author{
A. S. Al-Hobaib, M. S. Alsuhybani \& Kh. M. Al-Sheetan \\ Nuclear Science Research Institute, \\ King Abdulaziz City for Science and Technology (KACST), Saudi Arabia
}

\begin{abstract}
The present work is focused on developing a suitable chemical membrane with polyamide, incorporating co-solvents. There are many benefits of using a membrane technique. It is cost-effective and can be fabricated easily and its materials have less effect on the environment. Therefore, the general membrane techniques have been used for the desalination of ocean water as a worldwide strategy to meet the demand for clean water. But in some cases the use of this membrane becomes limited when pore size, distribution of pores and low selectivity for pollutants/contaminants are considered. The fabrication of such a membrane with co-solvents is expected to lead to a solution to address some of these problems. In this study, a polyamide thin-film composite membrane was developed by interfacial polymerization in non-polar heptane solvent, using acetone as a co-solvent medium. The modified membranes were characterized by different techniques. Scanning electron microscope and atomic force microscopy images showed a smooth membrane surface. Thermo-gravimetric analysis indicated that these developed membranes possessed high thermal stability. High contact angles were observed in the presence of acetone as a co-solvent in the polyamide membrane. Similarly, high fluxes were observed with low salt rejection ability.

Keywords: interfacial polymerization, RO membrane modified, co-solvent.
\end{abstract}




\section{Introduction}

Many physical-chemical methods have been developed and adopted at different times by researchers. Some use chemical treatments to remove these contaminants from the polluted water by adopting various techniques, while others use heat treatment for getting fresh water through vaporisation followed by condensation. These methods are not economically viable, time consuming and environmentally hazardous or unfriendly. But most significantly, some are using abundantly available ocean water to make fresh water through desalination process. In this desalination process many innovative techniques are continuously being developed. One of these techniques is the filtration of ocean water or decontamination of industrial effluents for reuse through membranes of different types. Thus, a great number of reverse osmosis (RO) and nanofiltration (NF) membranes with good desalination performance and permeate flux have been developed [1-4].

RO membrane is composed of three layers; bottom layer is made of unwoven polyester cloth having thickness of 100-200 $\mu \mathrm{m}$ to support the entire membrane; middle layer consists of polysulfone or polyethersulfone of thickness from $30-50 \mu \mathrm{m}$ and to support the top layer of polyamide or polyetherimide of average thickness from 100-200 nm, which is used to separate solutes from feed water. The polyamide membrane is prepared on the surface of the middle layer by interfacial polymerization between diamine moiety of 1,3-phenylenediamine (MPD) in the water phase and acid chloride moiety of 1,3,5-benzenetricarbonyl trichloride (TMC) in the non-polar organic phase. An industrial product named FT-30 is such a RO membrane, which has high level of salt rejection of more than $99 \%$ and flux of more than $1 \mathrm{~m}^{3} /\left(\mathrm{m}^{2} / \mathrm{d}\right)$ for $2000 \mathrm{ppm} \mathrm{NaCl}$ at $1.55 \mathrm{MPa}[5,6]$. Thus, many types of RO membranes were developed.

Incorporation of a co-solvent in an organic phase during the interfacial polymerization of MPD with TMC has been reported by many other researchers to bring about or cause structural and morphological changes in the RO membranes, Takashi Kamada developed polyamide RO membranes with controlled surface morphology by interfacial polymerization of water-soaked MPD with TMC in organic nonpolar hexane medium on PS ultrafiltration supports [7-12]. Co-solvent such as acetone was used in the organic phase in order to control both surface morphology and polyamide network matrix. He also used a synergistic co-solvent acetone $(2 \%)$ in the hexane phase in order to control polymerization reaction zone as well as to modify membrane network matrix [13]. This membrane showed selective molecular sieving of small molecules from larger ones. Addition of larger amount of acetone as a co-solvent in the hexane solution increased both pore size and water flux. RO membrane with 2 wt \% acetone showed rejection of more than $99.4 \%$ and high water transport at a rate of more than $1 \times 10^{-11} \mathrm{~m}^{3} /\left(\mathrm{m}^{2} \mathrm{~Pa} . \mathrm{s}\right)$. Polyamide membranes with controllable thin dense layer and effective nonporous were also fabricated by Kong et al. [13] by adding co-solvent acetone to nonpolar organic hexane phase by interfacial polymerization of MPD with TMC and this membrane exhibited a high water flux and salt rejection [13]. Addition of $2 \mathrm{wt} \%$ acetone as a co-solvent showed 
approximately 4 times higher water flux with no loss of salt rejection compared to that of membrane prepared without acetone.

In the present work, heptane will be used as a non-polar organic phase in place of hexane in the preparation of $\mathrm{RO}$ membrane by interfacial polymerization of MPD with TMC. This study will also investigate the effect of acetone as co-solvent on the performance of RO membrane.

\section{Experimental}

\subsection{Materials}

Various chemicals and reagents, which were used in this study, were of high purity.

\subsection{Preparation of membrane}

An commercial polysulfone (PS-20, Sepro) product named PS-20 was immersed in an aqueous solution of $2 \mathrm{wt} \%$ 1,3-phenylenediamine (MPD, >99\%, SigmaAldrich) for $2 \mathrm{~min}$. Then the excess MPD aqueous solution was removed by pressing under a rubber roller. The material was again immersed in $0.1 \mathrm{wt} \% 1,3,5-$ benzenetricarbonyl trichloride (TMC, $>98 \%$, Sigma-Aldrich) TMC/Heptane (99\%, Sigma-Aldrich) solution for $1 \mathrm{~min}$ in a variable quantity (e.g. 0.5, 1, 2, 3 and 5 wt. $\%)$ of acetone ( $>99 \%$, Sigma-Aldrich) as co-solvent. The product was then rinsed with $0.2 \mathrm{wt} \% \mathrm{Na}_{2} \mathrm{CO}_{3},(>99 \%$, Scharlau) washed with DI water and finally stored in a refrigerator at about $4{ }^{\circ} \mathrm{C}$ in DI water until use

\subsection{Characterization and instrumentation}

The morphology and microstructure of the as-synthesized nano-composite membrane was examined by means of a scanning electron microscope ((SEM), FEI Nova-Nano SEM-600, the Netherlands). Atomic force microscopy (AFM) was used to analyse the surface morphology and roughness of the prepared membranes. The AFM device was Nano surf scanning probe-optical microscope (Bruker Corporation). The thermal stability of the membranes with co-solvent concentration was characterized by thermo-gravimetric analysis (TGA) was performed using Pyris 1 (TGA from PerkinElmer USA), heating rate of $10^{\circ} \mathrm{C} / \mathrm{min}$ under nitrogen gas flow at $20 \mathrm{ml} / \mathrm{min}$. The temperature range was maintained from room temperature to $800^{\circ} \mathrm{C}$ using ceramic pan. FTIR-ATR spectroscopy with attenuated total reflection (ATR) plate (PerkinElmer USA). The spectra were measured over a wave number range of $4000-600 \mathrm{~cm}^{-1}$ at a resolution of $4 \mathrm{~cm}^{-1}$. Contact angle analysis was performed using a Ramé-Hart Model 250. The performance of the prepared membranes was analyzed through a cross-flow system (CF042SS316 Cell, Sterlitech Corp. USA). The valid membrane area in this system was $42 \mathrm{~cm}^{2}$. The Feed water temperature was $25^{\circ} \mathrm{C}$ with $\mathrm{pH}$ adjusted between 6 and 7, $2000 \mathrm{ppm}$ feed $\mathrm{NaCl}$ and 1 gallons per minute (gpm) feed flow rate. The filtration was carried out at the pressure of $225 \mathrm{psi}$. All measurements of 
the water flux and salt rejection were measured after 30 min of water filtration experiments to ensure that had reached stability. A schematic diagram of the crossflow filtration system is shown in figure 1.

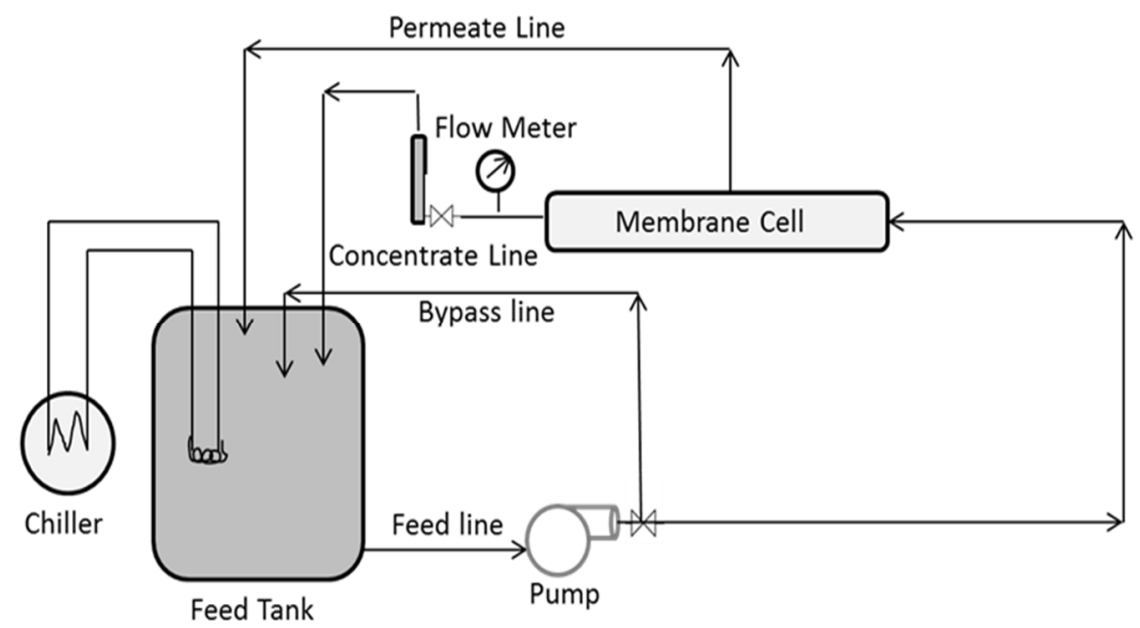

Figure 1: Schematic illustration of forward cross-flow filtration system.

The flux was calculated from the following eq. (1) [7]

$$
J=\frac{V_{p}}{A^{*} t}
$$

where $J$ is the water flux $\left(\mathrm{L} / \mathrm{m}^{2} \mathrm{~h}\right), \mathrm{Vp}$ is the permeate volume $(\mathrm{L}), \mathrm{A}$ is the membrane area $\left(\mathrm{m}^{2}\right)$ and $\mathrm{t}$ is the treatment time $(\mathrm{h})$. The salt rejection $(\mathrm{R})$ was calculated using the following eqn (2)

$$
R=\left(1-\frac{C_{p}}{C_{f}}\right) * 100
$$

where $C_{p}$ and $C_{f}$ are the salt concentrations of permeate and feed, respectively.

\section{Results and discussion}

\subsection{SEM analysis}

SEM images were taken for Polysulfone and TFC-reference are shown in figure 2 , respectively. The images of the membrane show that the surfaces are smooth homogeneous. 

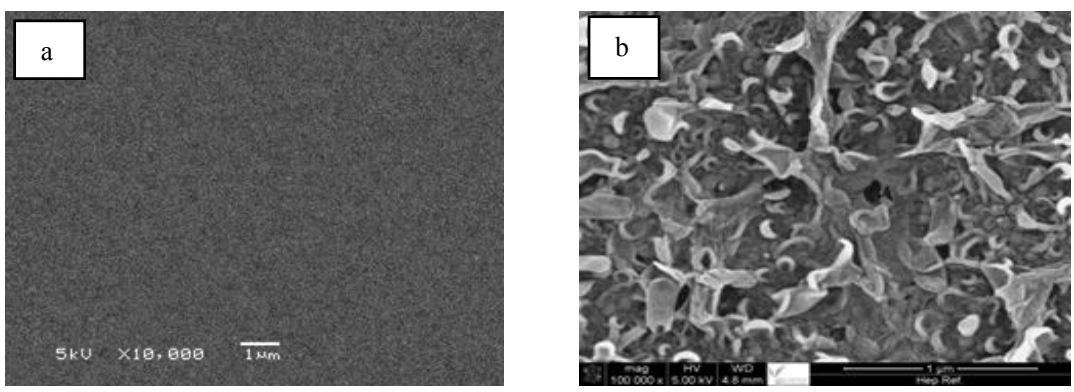

Figure 2: SEM images of PS membrane (a) and TFC reference (b).

SEM images of the membrane upon adding acetone in the non-polar organic phase shows effect of the acetone concentration on the morphology of the formed membranes, as depicted in figure 3. It is observed here that our results are in agreement with those results obtained by other researchers [12-18]. Moreover, as the concentration of a co-solvent increases, the pore size of the membranes formed gets larger.

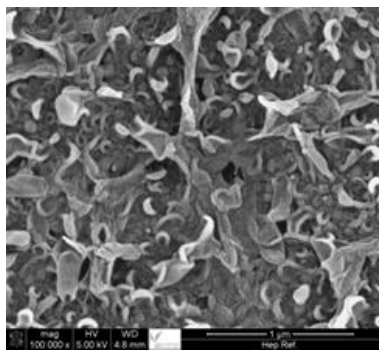

No additive

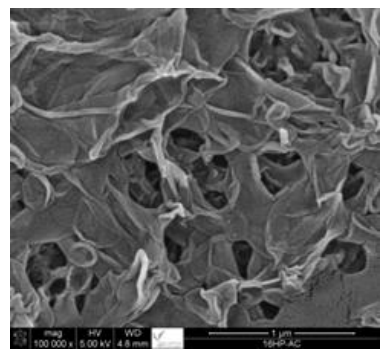

Acetone Conc. 0.5 wt $\%$

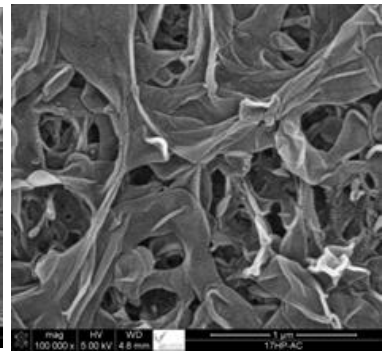

Acetone Conc. $1 \mathrm{wt} \%$

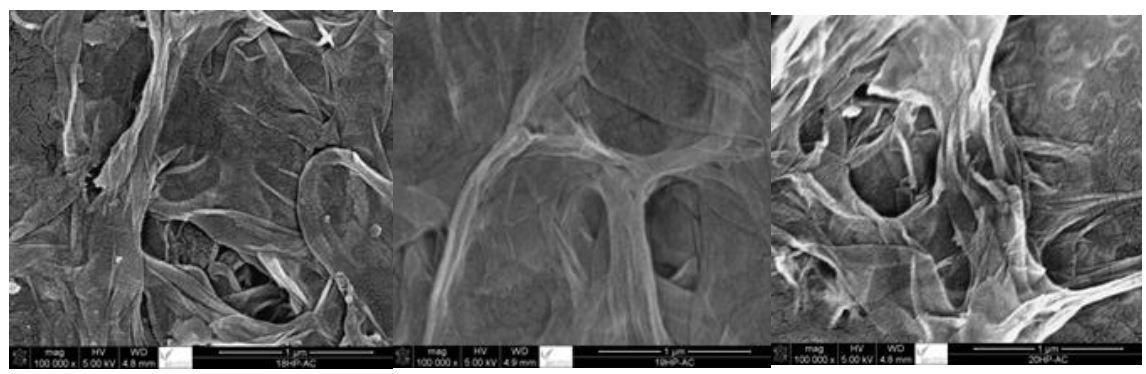

Acetone Conc. 2 wt $\%$

Acetone Conc. 3 wt $\%$

Acetone Conc. 5 wt $\%$

Figure 3: SEM images of the formulated membranes upon adding Acetone as co-solvents and effect the Acetone on the morphology of the membrane formed. 


\subsection{Atomic force microscope (AFM) analysis}

AFM images of the developed membranes are shown in figure 4. It is observed here that roughness has been produced in the surface of the membranes ranging between 27 and $33 \mathrm{~nm}$. The surface is formed of continuous ridge and valley structures confirming previous results obtained by researchers [14-18]. On the other hand, the flux of feed water solution through different membranes and the salt rejection data clearly indicate that each one of these membranes has different average pore size.

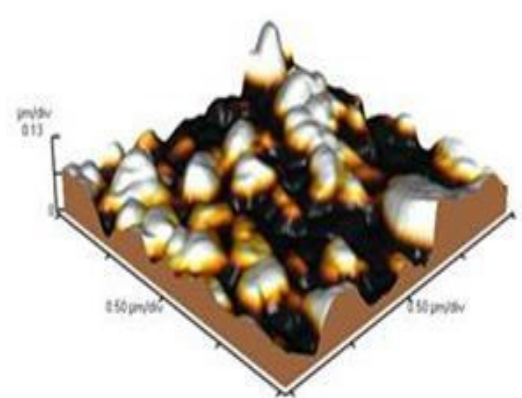

TFC reference, $\mathrm{Ra}=33 \mathrm{~nm}$

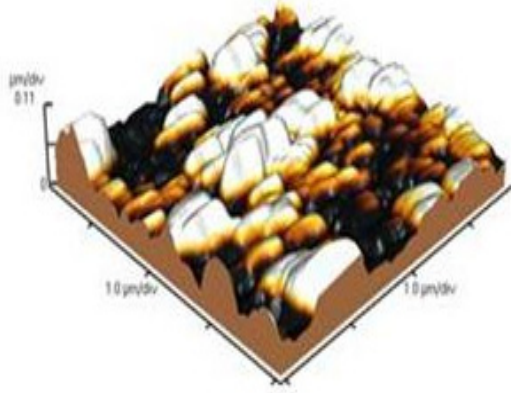

Conc. of Acetone $2 \mathrm{wt} \%, \mathrm{Ra}=27 \mathrm{~nm}$

Figure 4: AFM images of different membranes.

\subsection{Thermo-gravimetric analysis (TGA)}

Figure 5 shows thermal degradation behaviour of different membranes developed with PA on PS platform using different concentrations of acetone as co-solvent. It can be seen from figure 5 that both PS and PA membrane go through thermal decomposition in two main steps. The first step was in the temperature regions from $400-500^{\circ} \mathrm{C}$. The second step of degradation occurred between $550^{\circ} \mathrm{C}$ and $600^{\circ} \mathrm{C}$. This result is in good agreement with results reported by others [7-9]. However, a small step of degradation is noticed in the region of $100-170^{\circ} \mathrm{C}$ for PS. Results of degradation peaks, weight loss and residual mass derived from figure 5 of different membranes formed in the presence of various quantities of acetone are shown in table 2 . These results indicate that all membranes have high thermal stability with their degradation temperatures of about $481 \pm 2{ }^{\circ} \mathrm{C}$. This could be attributed to the sulfonic group in chains of polymer. The findings seem to be consistent with other researcher [10]. However, the second step of degradation occurs at around $580 \pm 3^{\circ} \mathrm{C}$. It is due to polymerization process and cross-linking processes during formation of the polyamide membrane [11]. Table 2 shows that residual weights of all samples were taken at $800^{\circ} \mathrm{C}$. It was from 20 to $22.07 \%$ depending on the concentration of acetone. 


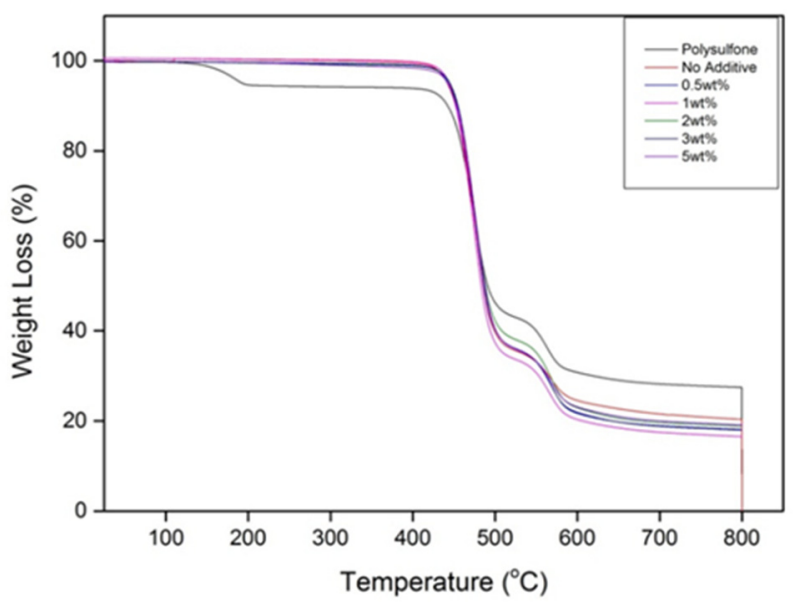

Figure 5: TGA results obtained for membranes.

Table 1: TGA values obtained from membranes.

\begin{tabular}{|c|c|c|c|c|}
\hline \multirow[t]{2}{*}{ Code } & \multirow[t]{2}{*}{ Frist peak } & \multirow{2}{*}{$\begin{array}{l}\text { Second } \\
\text { Peak }\end{array}$} & $\begin{array}{l}\text { Weight } \\
\text { Lose } \%\end{array}$ & $\begin{array}{c}\text { Residual } \\
\text { mass }\end{array}$ \\
\hline & & & \multicolumn{2}{|c|}{ at $800^{\circ} \mathrm{C}$} \\
\hline Polysulfone & 479.9 & 576.3 & 72.3 & 27.7 \\
\hline TFC-reference & 483.11 & 581.89 & 79.23 & 20.77 \\
\hline $0.5 \mathrm{wt} \%$ of acetone & 483.46 & 582.89 & 78.3 & 21.7 \\
\hline $1.0 \mathrm{wt} \%$ of acetone & 481.51 & 581.06 & 79.34 & 20.66 \\
\hline $2.0 \mathrm{wt} \%$ of acetone & 482.33 & 584.26 & 80 & 20 \\
\hline $3.0 \mathrm{wt} \%$ of acetone & 481.98 & 581.65 & 78.49 & 21.51 \\
\hline $5.0 \mathrm{wt} \%$ of acetone & 481.35 & 579.1 & 78.98 & 21.02 \\
\hline
\end{tabular}

\subsection{Fourier transmission infra-red (FTIR) analysis}

FT-IR spectra were taken from wavenumber 600 to $4000 \mathrm{~cm}^{-1}$ for developed membranes containing acetone as co-solvent is shown in figure 6 . The effect of acetone concentrations is also observed on these spectra. This means transmission $(\%)$ decreases with increase of concentration. However, transmission (\%) of light is higher for membranes made without co-solvent. Moreover, as the concentration of acetone increases the absorption of the light energy increases as well indicating the formation of thicker membrane layer. It is observed that at wavenumber $\sim 780$ $\mathrm{cm}^{-1}$, the $-\mathrm{CH}_{2}$-bond is influenced upon the addition of acetone. $\mathrm{A} \mathrm{C}=\mathrm{C}$ bond stretching appears at wavenumber of $1680 \mathrm{~cm}^{-1}$ for all membranes. 

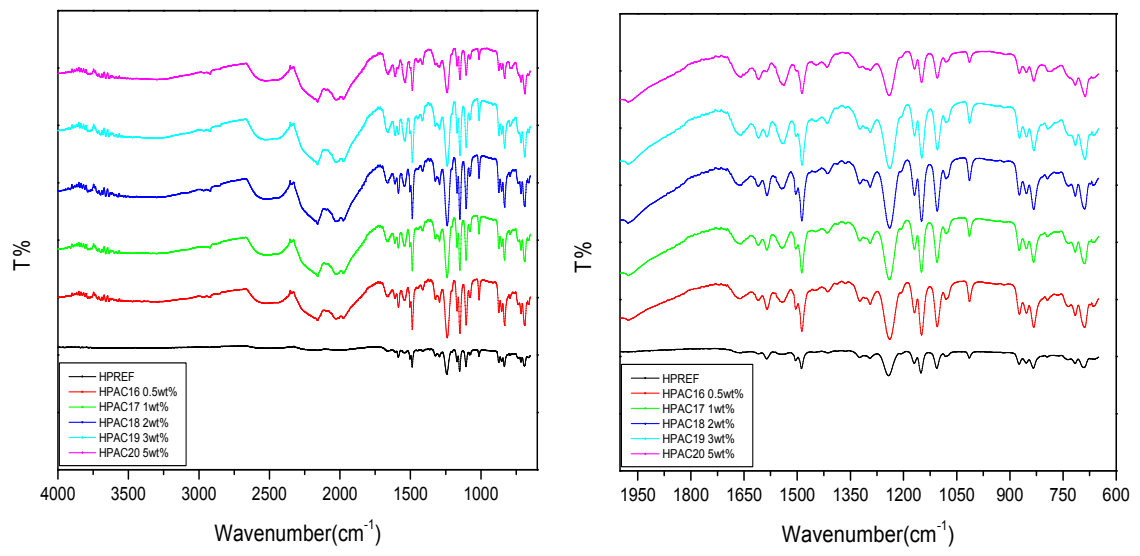

Figure 6: FT-IR spectra of membranes containing acetone as a co-solvent.

\subsection{Contact angle measurements}

Contact angles were measured with a droplet of DI water placed on a homogeneous surface of membranes prepared with acetone as co-solvents at different concentrations from 0.5 to $5 \mathrm{wt} \%$. A result is shown in figure 7 as a function of concentration. It is observed that contact angle significantly increases as concentration of acetone increases, reaches a peak of $2 \mathrm{wt} \%$ for all and then decreases with increase of concentration of acetone.

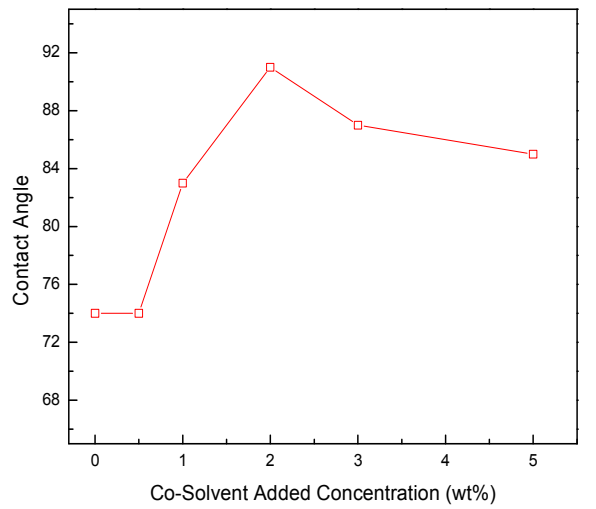

Figure 7: Contact angles as a function of concentrations of acetone as co-solvent.

\subsection{Membrane performance (flux and salt rejection):}

Isopropyl alcohol (IPA, 99.8\%, Scharlau), $\mathrm{NaCl}$ (99.5\%, Loba Chemie) salt and $\mathrm{MgCl}_{2}(99 \%$, Merck) solutions were used to measure permeate fluxes and salt 
rejection through different $\mathrm{PA}$ membranes containing acetone as co-solvent. Result is shown in figure 8: (A) for IPA, (B) for $\mathrm{NaCl}$ and (C) for $\mathrm{MgCl}_{2}$, respectively. Results clearly show that flux, in general, increases as the concentration of acetone increases. But the highest flux is obtained in the presence of acetone co-solvent indicating larger pore sizes. While the salt rejection of IPA, $\mathrm{NaCl}$ and $\mathrm{MgCl}_{2}$, that drops drastically at $2 \mathrm{wt} \%$. It is observed here that our results are in agreement with those results obtained by other researchers $[12,13,17,18]$.

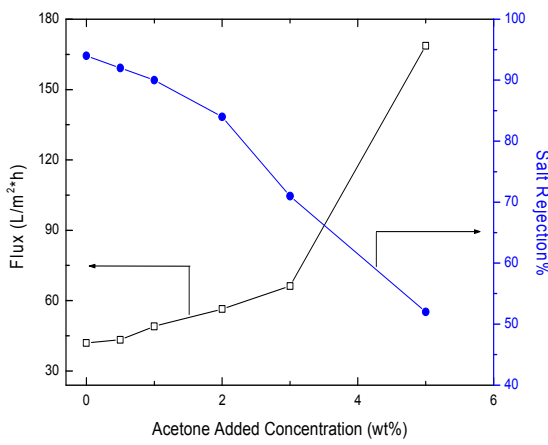

A) Flux and Salt rejection of IPA

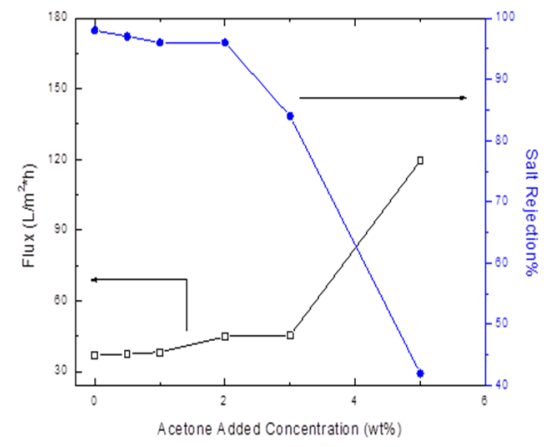

B) Flux and Salt rejection of $\mathrm{NaCl}$ solutions

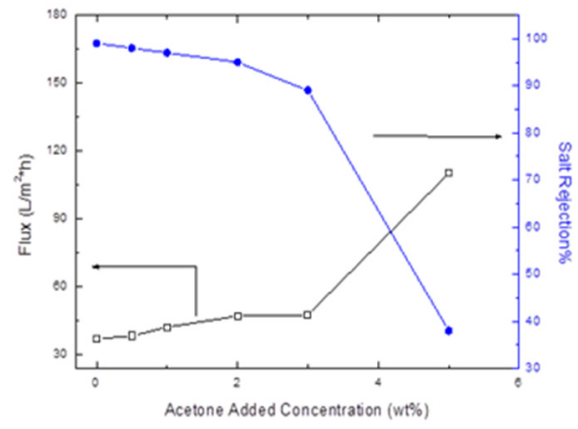

C) Flux and Salt rejection of aqueous $\mathrm{MgCl} 2$ solution

Figure 8: Flux and Salt rejection through membranes containing acetone as co-solvents.

\section{Conclusions}

Polyamide thin film composite membrane was developed by interfacial polymerization in non-polar heptane solvent with acetone as co-solvents medium. SEM images showed smooth membrane surface and average roughness of the surface was from 27 to $33 \mathrm{~nm}$. TGA analysis indicated that the developed 
membranes have high thermal stability. High contact angles were observed in the presence of acetone as a co-solvent in the PA membrane. Similarly, high fluxes were observed with PA membranes with low salt rejection ability.

\section{Acknowledgement}

Authors are grateful to King Abdulaziz City for Science and Technology, Riyadh, Saudi Arabia, for the financial support of this work and the facilities in its labs.

\section{References}

[1] Peng X.S., Jin J., Nakamura Y., Ohno T., Ichinose L., Ultrafast permeation of water through protein-based membranes, Nature Nanotechnol. 4 (2009) 353.

[2] Baker R.W., Membrane Technology and Applications, Second Edition, John Wiley \& Sons, Ltd, England, 2004.

[3] Kurihara M., Hanakawa M., "Megaton Water System: Japanese national research and development project on seawater desalination and waste water reclamation", Desalination, 308 (2013) 131-137.

[4] Xie W., Geise G.M., Freeman B.D., Lee H.S., Byun G., McGrath J.E., "Polyamide interfacial composite membranes prepared from m-phenylene diamine, trimesoyl chloride and a new disulfonated diamine", J. Membr. Sci. 403-404 (2012) 152-161.

[5] Petersen R.J, "Composite reverse osmosis and nanofiltration membranes", J. Membr. Sci. 83 (1993) 81-150.

[6] Cadotte J.E., "Interfacially synthesized reverse osmosis membrane", U.S. Patent 4, 277, 344, 1981.

[7] Lind, M. L.; Eumine Suk, D.; Nguyen, T.-V.; Hoek, E. M. V. "Tailoring the Structure of Thin Film Nanocomposite Membranes to Achieve Seawater RO Membrane Performance". Environ. Sci. Technol. 2010, 44, 8230-8235.

[8] Yu, S.; Liu, X.; Liu, J.; Wu, D.; Liu, M.; Gao, C. "Surface Modification of Thin-Film Composite Polyamide Reverse Osmosis Membranes with Thermo-Responsive Polymer (TRP) for Improved Fouling Resistance and Cleaning Efficiency”. Sep. Purif. Technol. 2011, 76, 283-291.

[9] Mohan, D. J.; Kullová, L. A "Study on the Relationship between Preparation Condition and Properties/performance of Polyamide TFC Membrane by IR, DSC, TGA, and SEM Techniques". Desalin. Water Treat. 2012, 51, 586-596.

[10] Guan, R.; Zou, H.; Lu, D.; Gong, C.; Liu, Y. "Polyethersulfone Sulfonated by Chlorosulfonic Acid and Its Membrane Characteristics. Eur. Polym. J. 2005, 41, 1554-1560.

[11] Zuo, J.; Chung, T.-S. "Design and Synthesis of a Fluoro-Silane Amine Monomer for Novel Thin Film Composite Membranes to Dehydrate Ethanol via Pervaporation”. J. Mater. Chem. A 2013, 1, 9814-9826. 
[12] Kamada T., Ohara T, Shintani T. and Tsuru T. "Optimizing the preparation of multi-layered polyamide membrane via the addion of a co-solvent"; J. Membrane Sci., 2014, 453, 489-497.

[13] Kong C., Shintani T., Kamada T., Freger V. and Tsuru T.; "Co-solvent mediated synthesis of thin polyamide membrane" J. Membrane Sci., 2011, 384, 10-16.

[14] Byeong-Heon Jeong, Eric M.V. Hoeka, Yushan Yan, Arun Subramani, "Interfacial polymerization of thin film nanocomposites: A new concept for reverse osmosis membranes", Journal of Membrane Science, (2007), 294, $1-7$.

[15] Chunlong Kong, Masakoto Kanezashi, Tetsuya Yamomoto, Takuji Shintani, "Toshinori Tsuru, Controlled synthesis of high performance polyamide membrane with thin dense layer for water desalination", Journal of Membrane Science, 2010, 362, 76-80.

[16] Chunlong Konga, Takuji Shintanib, Takashi Kamada, Viatcheslav Fregerc, Toshinori Tsur, "Co-solvent-mediated synthesis of thin polyamide membranes", Journal of Membrane Science, (2011), 384, 10-1.

[17] Takashi Kamada, Tomomi Ohara, Takuji Shintani, Toshinori Tsuru, "Controlled surface morphology of polyamide membranes via the addition of co-solvent for improved permeate flux" Journal of Membrane Science, (2014), 467, 303-312.

[18] Al-Hobaib, A. S. Alsuhybani, M. S. and AL-Sheetan, Kh. Mousa, M Hasan." Modification of a reverse osmosis membrane with co-solvents to improve performance" submitted to Membrane Science Journal (12/2014). 